\title{
Comparing Platelet-Rich Plasma Injections with Extracorporeal Shock Wave Therapy for Chronic Plantar Fasciitis: A Prospective Comparative Study
}

\section{Xin Wang}

Hebei Medical University Third Affiliated Hospital

Dong Zhang

Winter sports management center of Hebei province

\section{Fengqi Zhang}

Hebei Medical University Third Affiliated Hospital

Lin Jin

Hebei Medical University Third Affiliated Hospital

\section{Donglin Shi}

Hebei Sport university

\section{Zhiyong Hou ( $\nabla$ kobe20201124@163.com )}

Hebei Medical University Third Affiliated Hospital https://orcid.org/0000-0002-7667-9991

\section{Research article}

Keywords: Platelet-rich Plasma, Extracorporeal Shock Wave Therapy, Plantar Fasciitis, Treatment.

Posted Date: August 9th, 2021

DOI: https://doi.org/10.21203/rs.3.rs-718755/v1

License: (c) (1) This work is licensed under a Creative Commons Attribution 4.0 International License. Read Full License 


\section{Abstract}

\section{Background}

Plantar fasciitis is a self-limiting disease that is a common cause of adult heel pain. The purpose of this prospective study was to compare the curative effect following platelet-rich plasma (PRP) injections with extracorporeal shock wave therapy (ESWT), in the patients with chronic plantar fasciitis.

\section{Method:}

100 patients with plantar fasciitis were randomly divided into 2 groups (group A and group B), 50 patients in each group. Patients were treated with autologous PRP injection in group A and with dolorclast radial ESWT in group B. Outcomes were assessed prior to the therapy and at 3,6,12, and 24 months following the therapy, which included American Orthopaedic Foot and Ankle Society (AOFAS) scores, visual analog scale (VAS) pain scores, subjective satisfaction score and MRI studies were obtained in all cases to confirm the diagnosis of plantar fasciitis.

\section{Result}

No severe complications were recorded in the A and B groups. And in the treatment of plantar fasciitis have achieved good results. There was no significant difference in AOFAS scores between the two groups at 3 months after treatment. The AOFAS scores at 3,6, 12, and 24 months after treatment in group A were $91,85,84$, and 80 . The AOFAS scores in group B were $89,85,83$, and 79 , respectively. Both groups of patients had significant improvement compared with before treatment $(P<0.01)$.

\section{Conclusion}

The observations in this study indicate that PRP and ESWT are safe and feasible options for the treatment of chronic plantar fasciitis. But the long-term effect is not particularly satisfying.

\section{Introduction}

Plantar fasciitis (PF) is a self-limiting disease that is a common cause of adult heel pain.[1] The 40-70 year old middle-aged and elderly are high incidence groups.[2] The main reason is that changes in biomechanics and variations in the structure of the foot lead to inflammation of the plantar fascia and degeneration of the fascia.[3, 4, 5] However, sedentary, long-term standing, exercise overload, and obesity $\left(\mathrm{BM} \otimes 30 \mathrm{Kg} / \mathrm{m}^{2}\right)$ are common predisposing factors.[6, 7] The clinical manifestations of PF are mainly pain and discomfort in the heel, usually with tenderness at the center of the plantar. It is characterized by severe pain and swelling in the heel when the patient takes the first step in the morning or stays still for a long time. The symptoms are alleviated as the activity increases, but as the walking distance or standing time increases, the pain symptoms worsen.[3] 
PF can be treated by numerous methods, the main methods include conservative and surgical treatment. But there is no cure. The conservative treatment involves reduced or no weight bearing, nonsteroidal antiinflammatory drugs (NSAIDs), stretching the gastrocnemius and soleus muscles and the plantar fascia, orthopedic insoles, corticosteroids, extracorporeal shock wave therapy(ESWT), platelet-rich plasma (PRP) injections, are mainly aimed to reduce inflammation. $[8,9]$ while continued problems may require surgical intervention, however, it is reported that only $10 \%$ of patients undergo surgery.[10] The surgical treatment includes soft tissue release, plantar nerve release, calcaneus osteotomy and calcaneus spur resection.[11, 12] However, the surgery trauma is large, the price is high, and the postoperative effect is general, so the surgical treatment is not worthy of clinical promotion.

Although there are so many treatments for the PF, but there is currently no gold standard. The mechanism by which NSAIDs treat PF is to produce anti-inflammatory and analgesic effects by inhibiting cyclooxygenase (COX) and preventing the synthesis of prostaglandins (PGs). However, long-term use of large amounts of NSAIDs can cause adverse effects such as gastric mucosal damage, gastric bleeding, and even gastric perforation.[13] There are many types of insoles for the treatment of plantar fasciitis. The function of the insole is to reduce the pressure of the heel by providing shock absorbing cushion, while providing support for the medial arch to reduce the pressure on the plantar fascia.

The fascia of the fascia and the calcaneus at the stop point of the calcaneus can be evenly distributed. However, this method lacks long-term follow-up or the sample size is small, and further research is needed.[14] Corticosteroid injections are usually reserved for resistant PF after a conservative, noninvasive intervention failure. The potent anti-inflammatory effects of corticosteroids accelerate the process of pain relief. However, its use has also described complications such as application site infection, heel fat pad atrophy and plantar fascia rupture.

Corticosteroid injections are often reserved for resistant PF after failure of conservative noninvasive interventions. The potent anti-inflammatory effect of corticosteroids may hasten the process of pain relief. However, complications have also been described with its use, such as application site infections, heel fat pad atrophy, and plantar fascia rupture $[15,16,17]$.

At present, the literature reports that ESWT and PRP have achieved good results in the treatment of PF. ESWT can cause changes in substance $P$ after acting on bone and muscle tissue. Substance $P$ is a neuropeptide distributed in the nerve fibers. It is related to pain transmission. ESWT can release substance $P$ in a short time and reduce its concentration in nerve fibers, thus relieving pain.[18] Plateletpich plasma (PRP) is a plasma containing high concentrations of platelets obtained by centrifugation of autologous whole blood. It has been confirmed that PRP contains various growth factors such as platelet-derived growth factor (PDGF), transforming growth factor- $\beta_{1}$ (TGF $\beta_{1}$ ), and transforming growth factor- $\beta_{2}$, (TGF- $\left.\beta_{2}\right)$, insulin-like growth factor (IGF), epidermal growth factor (EGF) and vascular endothelial growth factor (VEGF), etc. These growth factors play an important role in tissue repair and regeneration $[19,20]$. 
Although the fullness of literature of studies focused on the conservative treatment of PF, there are no paper comparing the long-term efficacy and the clinical outcome of the ESWT and PRP. This prospective randomized study was conducted to compare the efficacy of ESWT and PRP in the treatment of plantar fasciitis with a minimum of 24-months follow-up.

\section{Methods}

A prospective cohort study of all patients undergoing PRP or ESWT for PF was completed. The study was conducted over a period of 2 years from January 2015 to September 2016. The protocol was approved by the local ethics committee. All patients provided informed written consent prior to treatment and study participation.

Inclusion criteria: History of PF for more than 6 months; The obvious symptoms of plantar fascia (the patients have pain and swelling in the heel when they takes the first step in the morning or stays still for a long time); Evidence of PF on X radiographic and MRI examination; Signing informed consent and agree to review on time.

Exclusion criteria: Any previous local injection treatment for heel pain; Heel surgery history (Ankle fracture, talar fracture, calcaneal fracture, etc.); Foot deformity (flatfoot, clubfoot, tarsal coalition, etc.); Contraindications to this surgery included congenital collagen deficiency, BMI $\varangle 30 \mathrm{Kg} / \mathrm{m} 2$, severe heart disease, lesions affecting liver and kidney function, severe diabetes, central nervous system diseases, other medical diseases and pregnant or lactating women.

100 patients with plantar fasciitis were randomly divided into 2 groups (group A and group B) , 50 patients in each group. Patients were treated with autologous PRP injection in group $A$ and with dolorclast radial ESWT in group $B$.

The group A (PRP) $₫$ Twenty five of the 50 patients (50\%) were male, and twenty-five (50\%) were female. The average age for therapy was $48.8 \pm 11.3$ (range, 33-70) years. Median follow-up duration was 28.2 (range, 24-35) months. The group B (ESWT): There were equal number of male and female patients in the $B$ group $₫ 25$ each. Average age was $44.9 \pm 9.6$ (range, 32-68) years. The average follow-up time was 27.2 (range, 24-35) months. No differences were found between the demographic characteristics of the two different treatment groups $(P>0.05)$.

All patients were screened with plain radiographs and MRI to confirm the diagnosis of plantar fasciitis. Outcomes were assessed prior to the therapy and at 3,6,12, and 24 months following the therapy, which included Orthopaedic Foot and Ankle Society (AOFAS) scores, visual analog scale (VAS) pain scores, subjective satisfaction score.

PRPखUnder aseptic precautions, $27 \mathrm{~mL}$ of the patient's peripheral whole blood was obtained using an 18gauge needle. Then, $3 \mathrm{~mL}$ ACD-A was added to the collected blood (in ratio of 1:9), and around $3 \mathrm{~mL}$ PRP was extracted by a double centrifugation technique at $1300 \mathrm{rpm}$ for 10 minutes to separate erythrocytes 
and then again at $3500 \mathrm{rpm}$ for 10 minutes to concentrate platelets using the Remi R-8C DX Laboratory Centrifuge (Remi Electrotechnik Ltd, Maharashtra, India).

The injection was given under strict aseptic precautions. The area to be injected was prepared with $10 \%$ povidone iodine scrub and draped with sterile towels. Injection was given by palpating the maximum tender point of heel with a medial approach with the patient in a prone position and ankle in a neutral position.

ESWT $₫$ Shockwave intervention was performed using an orthopedic dedicated divergent extracorporeal shock wave therapy machine (Switzerland, ENS). The patient takes a sitting position, and under nonanesthesia, according to the patient's pain point positioning (if the patient cannot be accurately positioned, it is positioned under the X-ray or B-ultrasound); the treatment site is determined, the coupling agent is applied to the treatment site, and the shock wave probe is placed at this time. position. Each impact is 2000 times ( $8 \mathrm{~Hz} 2.0 \sim 3.0$ bar), 7 days for 1 cycle, 4 to 8 times for 1 course of treatment; try to reduce strenuous exercise after treatment.

\section{EFFICACY EVALUTION CRITERIA}

Patient satisfaction evaluation of treatment outcomes mainly includes 'excellent', 'good', 'fair', or 'poor'. (Table 1) Outcomes were assessed prior to the therapy and at 3, 6,12, and 24 months following the therapy, which included Orthopaedic Foot and Ankle Society (AOFAS) scores, visual analog scale (VAS) pain scores and MRI studies.

\section{STATISTICAL ANALYSIS}

The statistical analysis was performed using the data analysis program SPSS Statistics version 16.0 software SPSS Inc ,Chicago, Illinois, USA). Nonnormally distributed continuous variables were compared using the Kolmogorov-Smirnov test. For all statistical tests. The paired t-test and Wilcoxon's signed-rank test were used to compare pre- and post-therapy values (AOFAS ankle-hindfoot scores, VAS scores,

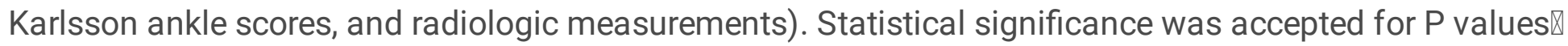
0.05 .

\section{Result}

At the time of final follow-up, all 100 patients (Group A and Group B) $(100 \%)$ returned for final evaluation. The first month after therapy, group A (PRP): Overall patient satisfaction was rated as excellent by 30 (60\%) patients, good by $17(34 \%)$ patients, fair by $1(2 \%)$ patient, and poor by $2(4 \%)$ patients. As time goes on, the patient's satisfaction rate is gradually reduced. After 2 years of treatment, most patients are followed by fair. and the number of excellent, good, and poor is not much different. It showed that 45 $(90 \%)$ patients rated the outcome good to excellent after 1 month of ESWT, the number of patients in fair and poor is 5 . the excellent and good decreased to 9 and 13, the fair and poor increased to 20 and 8 
respectively, at the end of 24 months; No statistically significant compared with group A ( $P \otimes 0.05)$. (Table 2)

In the PRP group, one patient who was dissatisfied with the result had the history of bone hyperplasia, calcaneal spur. The cause of another poor patient was unknown, and there was no significant relief after treatment. Compared with all other patients, the 52-year-old patient had a smoking history of 36 years. In the PRP group, one patient who was dissatisfied with the result had the history of bone hyperplasia, calcaneal spur. The cause of another poor patient was unknown, and there was no significant relief after treatment. Compared with all other patients, the 52-year-old patient had a smoking history of 36 years. Two of the poor patients in the ESWT group developed swelling of the plantar after treatment and the pain worsened after treatment, but the symptoms resolved on the fourth day.

At the time of final follow-up, the VAS of the two groups was significantly lower than that before the treatment, but the statistics were not significant between the two groups (P凶0.05). As the follow-up time increasing, VAS scores have increased to varying degrees. The mean pre- AOFAS score of PRP group (54.5 \pm 4.6$)$ improved significantly to a mean post- score of $92.8 \pm 3.6$ at the first month. Similarly, the patients in Group B have also improved significantly. Group A: AOFAS scores at 3, 6, 12, and 24 months after treatment were $91.1 \pm 2.2,85.7 \pm 3.8,84.4 \pm 2.1$, and $80.8 \pm 3.5$, respectively. The score gradually decreased, but the effect was significant compared with before treatment. It is statistically significant. (Table 3 Table 4)

\section{Discussion}

Various conservative measures have been proposed as suitable alternatives to surgical intervention for PF. Unfortunately, no guidelines are available for the treatment of PF. However, for many years, PRP and ESWT has been the effective treatment of patients with recalcitrant PF. The purpose of this prospective study was to compare the curative effect following PRP injections with ESWT, in the patients with chronic plantar fasciitis.

In order to ensure the reliability of the research, PRP should avoid various influencing factors in the preparation process. Among them, platelets themselves have physiological fluctuations, that is, different parts (venous, distal), different body positions (lying position, standing position), and before and after exercise, platelet counts are significantly different. Therefore, in this study, the patient was given blood in the same state, and the tourniquet was not taken, and the blood was collected with a needle of 18 gauge. Use plastic test tubes that are not easy to adsorb platelets, use ACD-A anticoagulant that protects platelets, and ensure that treatment is completed within 2 hours. Trying to reduce the interference factor.

However, with the extension of follow-up time, the subjective satisfaction of patients decreased, and the VAS score and AOFAS score also showed different degrees of change. The satisfaction rate was $90 \%$ (Excellent 38\%, Good 52\%) at 3 months after treatment, and the patient satisfaction rate was $22 \%$ (Excellent 18\%, Good 26\%) at the last follow-up. There was statistically significant difference pre- and post- treatment (Pख0.05). It shows that the efficacy of PRP in the treatment of PF in a short period of time 
is very obvious. With the prolongation of time, the effect will subside, and it has a certain relationship with the patient itself, but the specific reasons are waiting for the follow-up study.

ESWT is a kind of mechanical wave with certain acoustic and mechanical physical properties, featuring high pressure, short cycle and high frequency.[21] With the deepening of research in recent years, extracorporeal shock wave therapy has been widely used in orthopedics.[22] When the shock wave enters the human body, different mechanical stress effects are generated at the interface of different tissues, which are manifested by different tensile stresses and compressive stresses on the tissue cells. The tensile stress can cause the release between the tissues and promote the microcirculation; the compressive stress can elastically deform the cells and increase the oxygen uptake of the cells. The cavitation effect is that the shock wave causes a large number of tiny bubbles in the tissue to rapidly expand, which is beneficial to clear the occluded microvessels and improve the microcirculation; and causes the release of growth factors, the reactivation of primitive stem cells, the activity of osteoblasts, and the regeneration of blood vessels and tissues. Promotes the biological effects of callus formation and bone healing $[23,24]$. Other scholars believe that pain relief is due to shock wave stimulation that increases the threshold level of diseased tendon pain. It may be due to the strong impact of extracorporeal shock waves on human tissues. Local high-intensity shock waves can cause excessive changes in pain-induced neurosensory receptors, release free radicals around the cells, release substances that inhibit pain, reduce neurological sensitivity, and block nerve conduction. Thereby the pain is alleviated $[25,26]$.

Several studies compared the effects of extracorporeal shock waves with surgery, topical corticosteroid injection, or physical therapy for the treatment of plantar fasciitis. Surgery and shock waves achieved the same effect.[27] However, shock waves have no surgical risk and surgical pain, physical therapy and shock wave effects. Quite, but physical therapy is time consuming and inconvenient. Hormone therapy has a good short-term effect, and the long-term effect shock wave is better. The changes in VAS and AOFAS scores before and after treatment in patients in the ESWT treatment group in this study also showed that the shock wave treatment of plantar fasciitis is effective.[28] However, with the prolongation of follow-up time, the same excellent rate appeared in the PRP group.

After a detailed follow-up study, of the 100 patients treated, 4 out of 14 patients with poor had no improvement after treatment. The remaining 10 patients worsened heel pain mainly because of increased physical activity after symptoms were alleviated or wearing stiff soles. Therefore, the patient's own factors are also the cause of the effect.

However, the article still has certain defects. First, further research will expand the number of cases. Secondly, the senior surgeon performed subjectively the clinical evaluations, introducing the possibility of evaluation bias. Also, the effects of different doses on plantar fasciitis were studied, and the changes of microcirculation, thickness and flexibility of the plantar fascia were observed. And further research is warranted to confirm the present findings. 


\section{Conclusion}

The observations in this study indicate that PRP and ESWT are safe and feasible options for the treatment of chronic plantar fasciitis. But the long-term effect is not particularly satisfying.

\section{A List Of Abbreviations}

Platelet-rich Plasma PRP

Extracorporeal Shock Wave Therapy ESWT

American Orthopaedic Foot and Ankle Society AOFAS

Visual Analog Scale VAS

Plantar Fasciitis PF

Nonsteroidal Anti-inflammatory Drugs NSAIDs

Cyclooxygenase COX

Prostaglandins PGs

Platelet-derived Growth Factor PDGF

Transforming Growth Factor- $\beta_{1}$ TGF $\beta_{1}$

Transforming Growth Factor- $\beta_{2}$ TGF- $\beta_{2}$

Insulin-like Growth Factor IGF

Epidermal Growth Factor EGF

Vascular Endothelial Growth Factor VEGF

\section{Declarations}

\section{ACKNOWLEDGMENT}

1.Youth Science and Technology of Health Commission of Hebei, grant number,20180425, and Special Project for Winter Olympics of Science and Technology of Hebei, grant number:19975708D.

2.Supported by the Innovation Ability Promotion Project of Hebei Province (No.19245709D) Research on Key Techniques of physical therapy and functional training for elite winter athletes. 
All authors confirm that this work was approved by our Institutional Review Board with ethical principles of research.

This work was performed at the Third Hospital of Hebei Medical University, Hebei, China.

\section{AVAILABILITY OF DATA AND MATERIALS}

The patients' data were collected in the Third Hospital of Hebei Medical University. The datasets used and/or analyzed during the current study are available from the corresponding author on reasonable request.

\section{ETHICS APPROVAL AND CONSENT TO PARTICIPATE}

This study was conducted with approval from the Ethics Committee of the Third Hospital of Hebei Medical University. Written informed consent to participate was obtained from all participants.

\section{COMPETING INTERESTS}

The authors declare that they have no competing interests.

\section{AUTHOR DETAILS}

1. The Third Hospital of Hebei Medical University

2. Winter sports management center of Hebei Province

3. Hebei Sport University

\section{References}

1. Worp HVD, Akkerscheek IVD, Schie HV, et al. ESWT for tendinopathy: technology and clinical implications[J]. Knee Surgery Sports Traumatology Arthroscopy. 2013;21(6):1451-8.

2. Ertuğrul Akşahin. The comparison of the effect of corticosteroids and platelet-rich plasma (PRP) for the treatment of plantar fasciitis.[J]. Archives of Orthopaedic \& Trauma Surgery, 2012, 132(6):pp. 781-5.

3. Jain SK, Suprashant K, Kumar S, et al. Comparison of Plantar Fasciitis Injected With Platelet-Rich Plasma vs Corticosteroids[J]. Foot \& Ankle International, 2018, 39(7):p. 780.

4. Pohl M, Hamill J, Davis IS. Biomechanical and anatomic factors associated with a history of plantar fasciitis in female runners. Clin Journal Sport Med. 2009;19(5):372-6.

5. Melegati G, Tornese D, Bandi M, Caserta A. The influence of local steroid injections, body weight and the length of symptoms in the treatment of painful subcalcaneal spurs with extracorporeal shock wave therapy. Clin Rehabil. 2002;16(7):789-94. 
6. Thomas JL, Christensen JC, Kravitz SR, et al. The diagnosis and treatment of heel pain: A clinical practice guideline-revision 2010. J Foot Ankle Surg. 2010;49:1.

7. Acosta-Olivo C, Elizondo-Rodriguez J, Lopez-Cavazos R, et al. Plantar fasciitis. A comparison of treatment with intralesional steroids versus platelet-rich plasma (PRP). A randomized, blinded study. [J]. Journal of the American Podiatric Medical Association; 2016.

8. Johnson RE, Haas K, Lindow K, et al. Plantar fasciitis: what is the diagnosis and treatment?[J]. Orthop Nurs. 2014;33(4):198.

9. Acosta-Olivo C, Elizondo-Rodriguez J, Lopez-Cavazos R, et al. Plantar fasciitis. A comparison of treatment with intralesional steroids versus platelet-rich plasma (PRP). A randomized, blinded study. [J]. Journal of the American Podiatric Medical Association; 2016.

10. Stasinopoulos D, Johnson MI. Effectiveness of extracorporeal shock wave therapy for tennis elbow (lateral epicondylitis). Br J Sports Med. 2005;39:132-6.

11. Toomey EP. Plantar heel pain. Foot Ankle Clin. 2009;14:229-45.

12. Cheung JT, et al. Consequences of partial and total plantar fascial release: a finite element study. Foot Ankle Int. 2006;27:125-32.

13. Warner TD, Giuliano F, Vojnovic I, et al Warner TD, et al. Nonsteroid drug selectivities for Cyclooxygenase-1 rather than cyclo- oxygenase-2 are associated with human gastrointestinal toxicity: A full in vitro analysis. Proc Natl Acad Sci U S A 96, 7563-7568[J]. Proceedings of the National Academy of Sciences, 1999, 96(13):7563-7568.

14. Lynch DM. Conservative treatment of plantar fasciitis: a prospective study. J Am Pod Med Assoc. 1998;88(8):375-80.

15. Acevedo JI, Beskin JL. Complications of plantar fascia rupture associated with corticosteroid injection. Foot Ankle Int. 1998;19(2):91-7.

16. Kiter E, Celikbas E, Akkaya S, Demirkan F, Kiliç BA. Comparison of injection modalities in the treatment of plantar heel pain: a randomized controlled trial. J Am Podiatr Med Assoc. 2006;96(4):293-6.

17. Sellman JR. Plantar fasciitis ruptura associated with corticosteroid injection. Foot Ankle Int. 1994;15:376.

18. Metzner G, Dohnalek C, Aigner E. High-energy Extracorporeal Shock-Wave Therapy (ESWT) for the treatment of chronic plantar fasciitis.[J]. Foot \& Ankle International, 2010, 31(9):pp. 790-6.

19. Weibrich G, Kleis WK, Hafner G. Growth factor levels in the platelet-rich plasma produced by 2 different methods: curasan-type PRP kit versus PCCS PRP system[J]. Int J Oral Maxillofac Implants. 2002;17(2):184-90.

20. Cenni E, Granchi D, Vancini M, et al. Platelet release of transforming growth factor-beta and betathromboglobulin after in vitro contact with acrylic bone cements.[J]. Biomaterials. 2002;23(6):147984. 
21. Gamba C, Sala-Pujals A, Perez-Prieto D, et al. Relationship of Plantar Fascia Thickness and Preoperative Pain, Function, and Quality of Life in Recalcitrant Plantar Fasciitis[J]. Foot \& Ankle International, 2018, 39(6):p. 107110071877204.

22. Frassanito P, Cavalieri $C$, Maestri R, et al. Effectiveness of extracorporeal shock wave therapy and kinesio taping in calcific tendinopathy of the shoulder: a randomized controlled trial.[J]. Eur J Phys Rehabil Med, 2017.

23. Akınoğlu B, Köse N. A comparison of the acute effects of radial extracorporeal shockwave therapy, ultrasound therapy, and exercise therapy in plantar fasciitis[J]. Journal of Exercise Rehabilitation. 2018;14(2):306-12.

24. Ching-Jen Wang M, Hsuan-Ying Huang M, Chun-Hwan Pai M. Shock wave-enhanced neovascularization at the tendon-bone junction: An experiment in dogs[J]. Journal of Foot Ankle Surgery. 2002;41(1):16-22.

25. Wang FS, Wang CJ, Huang HJ, et al. Physical shock wave mediates membrane hyperpolarization and Ras activation for osteogenesis in human bone marrow stromal cells[J]. Biochemical \& Biophysical Research Communications, 2001, 287(3):pp. 648-55.

26. Purcell RL, Schroeder IG, Keeling LE, et al. Clinical Outcomes After Extracorporeal Shock Wave Therapy for Chronic Plantar Fasciitis in a Predominantly Active Duty Population.[J]. Journal of Foot \& Ankle Surgery Official Publication of the American College of Foot \& Ankle Surgeons, 2018, 57(4).

27. Othman AM, Ragab EM. Endoscopic plantar fasciotomy versus extracorporeal shock wave therapy for treatment of chronic plantar fasciitis. Arch Orthop Trauma Surg. 2010;130:1343-7.

28. Porter MD, Shadbolt B. Intralesional corticosteroid injection versus extracorporeal shock wave therapy for plantar fasciopathy. Clin J Sport Med,2005. 15:119-124.

\section{Tables}

Due to technical limitations, table $1,2,3,4$ is only available as a download in the Supplemental Files section.

\section{Supplementary Files}

This is a list of supplementary files associated with this preprint. Click to download.

- Table1.jpg

- Table2.jpg

- Table3.jpg

- Table4.jpg 